\section{TOPOLOGICAL INSULATORS}

\section{Winding polaritons}

\section{Phys. Rev. X 5, 031001 (2015)}

Torsten Karzig and co-workers from the USA and Israel have proposed a type of polariton with a 'winding' phase in momentum space that could enable unidirectional propagation. In their theoretical and numerical study they investigate excitons at the edges of semiconductor systems embedded into a photonic waveguide, using quantum wells or transition metal dichalcogenides (TMDs) and a magnetic field. CdTe quantum wells are proposed as a specific experimental candidate as they enable strong exciton-photon coupling. Another alternative proposed by the authors is monolayers of TMDs. The team describes conditions under which such thin TMDs should offer $\sim 20$ meV exciton-photon coupling, which may enable similar topological gaps as CdTe quantum wells. It should be noted that strong magnetic fields $(1.5 \mathrm{~T})$ will be required to achieve such gaps with TMDs. The simulations by Karzig et al. show that when excited, the topological polaritonic edge states propagate chirally. Although immune from backscatter, exciton and photon losses lead to

\section{IMAGING}

\section{Ultrasonic lens}

\section{Nature Meth. 12, 759-762 (2015)}

Imaging techniques with high spatiotemporal resolution aid understanding of complex biological systems. Now, by integrating an optical phase-locked ultrasound lens into a laser-scanning two-photon fluorescence microscope, Lingjie Kong and colleagues from the USA have demonstrated volumetric imaging at tens of hertz. They basically insert an ultrasound lens between the pulse compressor and the scanning microscope. dissipation and decay over time.

Precisely driving the lens using a phase-locked loop near its $\sim 455 \mathrm{kHz}$ resonance provides a single axial scan in $\sim 1.1 \mu \mathrm{s}$, and integrating the lens into a two-photon microscope with galvo scanners achieves a $\sim 1 \mathrm{kHz}$ crosssectional frame rate. Depending on the axial scan ranges, $40 \mu \mathrm{m}$ and $130 \mu \mathrm{m}$, the team has achieved lateral and axial resolutions of $\sim 0.5-0.6 \mu \mathrm{m}$ and $2.9-4.6 \mu \mathrm{m}$, and $0.7-1.0 \mu \mathrm{m}$ and $7.8-15.3 \mu \mathrm{m}$, respectively. The system was successfully applied to multicolour imaging of different processes that are sensitive to motion artefacts, including calcium and cell dynamics, and leukocytes in blood flow. The researchers say that the system can be used to upgrade existing laser scanning microscopes and will be attractive for improved threedimensional biological imaging.

\section{$R W$}

\section{TERAHERTZ SPECTROSCOPY \\ Magneto measurements \\ Nature Phys. http://doi.org/6q3 (2015)}

Traditional measurement technology cannot unambiguously quantify the fundamental parameters of the magnetotransport of electrons (the spin-dependent density and momentum scattering time of conducting electrons at the Fermi level) because of the need to make assumptions. Now, Zuanming Jin and co-workers from Germany and Portugal have discovered that ultrafast terahertz spectroscopy can perform the task. Single-cycle terahertz pulses were sent to a sample featuring alternating layers of $\mathrm{NiCoFe}$ and $\mathrm{Cu}$ and the transmission was measured in the time domain under the external magnetic field. The key to success is that ultrafast terahertz spectroscopy is a contact-free conductivity sampling method that suppresses spin accumulation at interfaces and contacts, preserving the native spin structure of the sample during the measurement. The electron momentum scattering times for majority- and

\section{NONLINEAR OPTICS}

\section{Hybrid interference}

Phys. Rev. Lett. 115, 043602 (2015)

Linear optical elements, such as beamsplitters, are commonly used for setting up separate optical paths and recombining light in interferometers. Bing Chen and colleagues from China and the USA have now reported an interferometer in which Raman amplification replaces the traditional beamsplitting elements. Optical and atomic correlation is established through the Raman process, which enables high-contrast interference fringes that are particularly sensitive to changes in optical path length and atomic phase. The atomic phase can be adjusted by magnetic field or Stark shifts. Previous work on similar ideas was mainly sensitive only to the optical phase but here the atomic phase is involved. In the set-up used by Chen et al. an amplified Stokes beam is combined with a write beam in an atomic cell. The recombination delay is adjusted by a Sagnac loop, which also plays an important role in stabilizing the optical phase. The interference fringes resulting from atom-photon correlations may be used to interrogate atomic states and could offer phase measurement precision exceeding the standard quantum limit. minority-spin were determined to be $57 \mathrm{fs}$ and $19 \mathrm{fs}$, respectively. The corresponding values for sheet densities were $1.35 \times 10^{20} \mathrm{~m}^{-2}$ and $1.36 \times 10^{20} \mathrm{~m}^{-2}$, respectively.

Importantly, further analysis revealed that the spin-asymmetry parameters for electron scattering - a key parameter for the giant magnetoresistance effect - was 3.00 at $300 \mathrm{~K}$, which was much larger than expected from traditional measurements.

\section{OPTOMECHANICS}

\section{Artificial magnetic fields \\ Optica 2, 635-641 (2015)}

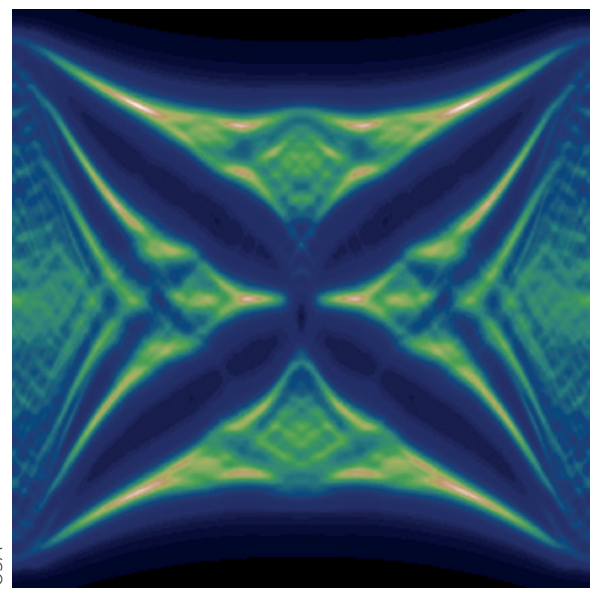

Optomechanical interactions in a photonic crystal lattice can be used to create artificial magnetic fields and non-reciprocal photon edge states that are immune to backscatter. That's the prediction of a theoretical investigation recently published by a collaboration between scientists at the University of Erlangen-Nürnberg in Germany and Caltech in the USA. The team says that by illuminating a patterned dielectric slab with two laser beams with suitably engineered optical phases and amplitudes it should be possible to induce optomechanical interactions that result in photon transitions that yield an arbitrary magnetic flux distribution. Two optomechanical approaches - modulated photon transitions and wavelength conversion - are considered by the team and their analysis suggests that both should be experimentally viable if fabrication-induced disorder can be reduced to a sufficiently low level. The scientists say that the attraction of the optomechanical approach to the creation of non-reciprocal states is that it is controlled all-optically rather than requiring electrodes or relying on the geometry of a structure and thus offers the opportunity for in situ tunability and great flexibility. 